\title{
Geometrization of Quantum Mechanics
}

\author{
José F. Cariñena ${ }^{\dagger}$, Jesús Clemente-Gallardo* and Giuseppe Marmo \\ †Departamento de Física Teórica, Universidad de Zaragoza, \\ 50009 Zaragoza, Spain. \\ `Instituto de Biocomputación y Física de los Sistemas complejos, \\ Universidad de Zaragoza, 50009 Zaragoza, Spain. \\ ${ }^{\ddagger}$ Dipartimento di Scienze Fisiche, Università Federico II di Napoli \\ and \\ INFN, Sezione di Napoli, Complesso Universitario di Monte Sant'Angelo \\ Via Cintia, 80126 Napoli, Italy
}

\begin{abstract}
We show that it is possible to represent various descriptions of Quantum Mechanics in geometrical terms. In particular we start with the space of observables and use the momentum map associated with the unitary group to provide an unified geometrical description for the different pictures of Quantum Mechanics. This construction provides an alternative to the usual GNS construction for pure states.
\end{abstract}

Keywords: Quantum Mechanics, Hermitian structure, Jordan algebra, Poisson bracket, Jordan-Lie algebra

\section{Introduction}

The description of any physical system, either classical or quantum, requires the identification of a space of states $\mathcal{S}$, a space of observables $\mathcal{O}$, and a pairing between both spaces with values in the field of real numbers $\mathbb{R}$. This pairing may be interpreted as the measurement process.

Usually in classical mechanics the space of states is given the structure of a real differentiable manifold, the space of observables is identified with the space of functions on the manifold and the pairing is the evaluation of a function at the state. Alternative approaches start with the associative and commutative algebra of functions (observables) and identify states as algebrahomomorphisms from the algebra of observables to that of real numbers. This 
approach has been advocated by Alexander Vinogradov in several papers and an account can be found in [1].

For quantum systems, following Schrödinger and Dirac, the primary object is the space of states $\mathcal{S}$. It is usually identified with a separable complex Hilbert space $\mathcal{H}$ [2]. Observables are a derived object and they are identified with self-adjoint operators acting on $\mathcal{H}$ (domain problems are not important at this stage). The pairing is provided by the expectation values of observables at elements of $\mathcal{H}$.

An alternative approach, which is a development of Heisenberg description of quantum mechanics in terms of 'infinite-dimensional matrices', mainly due to Segal [3], Haag and Kastler [4], starts with a $\mathbb{C}^{*}$-algebra and the observables (real dynamical variables) are given by its self-adjoint elements. States are then constructed as linear functionals on the algebra with further requirements to be able to interpret their evaluation on observables in terms of measurement processes.

In summary, it appears that while classical mechanics has a highly geometric content in its formulation, quantum mechanics is to be formulated mainly in terms of (linear) algebraic structures. While the relevant group of transformations of classical mechanics is hence a subgroup of the diffeomorphism group of the manifold of states (in the spirit of Klein's Erlangen Programme), the relevant group of quantum mechanics is a subgroup of linear transformations. However, it is commonly accepted that classical mechanics must be a suitable limit of quantum mechanics (this is usually known as the 'correspondence principle'). Therefore, it is reasonable to expect that a better understanding of this 'limit procedure' may be achieved if we elaborate a 'geometrization' of quantum mechanics in such a way that we might go beyond the group of linear transformations. Attempts at a geometrical formulation of Quantum Mechanics have been made in the past [5, 6, 7, 8, 9, 10, 11, 12, 13, 14, 15], but approaching the problem from a different perspective, namely by starting from the Hilbert space of states and trying to identify the necessary geometrical structures arising from the Hermitian structure.

The aim of this paper is to outline another way to achieve the geometrization. We shall follow an approach close to the $\mathbb{C}^{*}$-algebraic one we have mentioned earlier. To avoid technicalities which may obscure the mathematical geometric structures we are going to introduce, we shall limit ourselves to finite-level quantum systems. We hope that in this way the geometrical structures will emerge more neatly. 
Our primary object will be the family of Hermitian operators (observables) in $\mathcal{H}$, which is in a one-to-one correspondence with the real Lie algebra $\mathfrak{u}(\mathcal{H})$ of the unitary group $U(\mathcal{H})$. The real vector space $\mathfrak{u}(\mathcal{H})$ carries not only the structure of a Lie algebra, but also the structure of a Jordan algebra [16], and these two structures are compatible in a sense that will be clear later.

A 'geometrization' of the Lie algebra structure on the space of observables is easily achieved by replacing the Lie commutator with a linear Poisson tensor defined on the dual space $\mathfrak{u}^{*}(\mathcal{H})$ of the Lie algebra $\mathfrak{u}(\mathcal{H})$. In a similar manner we also geometrize the Jordan algebra structure defining the corresponding tensor on the same dual vector space. With this replacement we are not restricted to just linear transformations anymore, but both $\mathfrak{u}(\mathcal{H})$ and $\mathfrak{u}^{*}(\mathcal{H})$ are considered as $n^{2}$-dimensional manifolds.

Starting with the Poisson manifold structure on this space we may look for its symplectic realizations. In particular, a symplectic realization by means of a symplectic vector space replaces the more familiar Gelfand-NairmarkSegal (GNS) construction of the Hilbert space associated with the $\mathbb{C}^{*}$-algebra of dynamical variables starting from a pure state.

The symplectic vector space, equipped with an appropriate complex structure, will give rise to a Hilbert space $\mathcal{H}$. The symplectic action of the corresponding unitary group provides us with a momentum map $\mu: \mathcal{H} \rightarrow \mathfrak{u}^{*}(\mathcal{H})$ which defines the sought symplectic realization of the space of observables we were looking for. We relate also the space of pure states with the complex projective space which turns out to be symplectomorphic with minimal symplectic orbits of the corresponding coadjoint action (of the unitary group) on the space $\mathfrak{u}^{*}(\mathcal{H})$.

\section{The space of observables}

Following the algebraic approach advocated by Segal [3] and Haag and Kastler [4], we consider the space of observables as the collection of all the self-adjoint elements of a $\mathbb{C}^{*}$-algebra with identity element. The set of states $\mathcal{S}$ is then the collection of all positive real valued linear functionals $\Phi$, normalized by the condition

$$
\Phi(\mathbb{I})=1 .
$$

The pairing of both sets is provided by evaluation of the state, i.e. taking the expectation value of the observable when the state of the system is $\Phi$. 


\subsection{The algebraic structures}

There is also a well-known result that states that every $\mathbb{C}^{*}$-algebra with identity can be realized as the set of all bounded operators acting on some Hilbert space $\mathcal{H}$ (see [17] for details). Having decided to deal with finite-level quantum systems, we shall identify the set of observables with the space of Hermitian operators (i.e. those operators satisfying $A^{\dagger}=A$, condition which is now to be written as $A^{*}=A$ with the $\mathbb{C}^{*}$-involution). They define a real vector space isomorphic with the algebra $\mathfrak{u}(\mathcal{H})$, which corresponds to the operators which are anti-Hermitian and which, in the case of finite dimensional systems, define the Lie algebra $\mathfrak{u}(n), n$ being the dimension of the Hilbert space.

The multiplication by the imaginary unit $i$ establishes a vector space isomorphism with the real vector space of the Lie algebra of the unitary $\operatorname{group} \mathfrak{u}(\mathcal{H})$

$$
\alpha: \mathcal{O} \rightarrow \mathfrak{u}(\mathcal{H}) \quad \alpha(A)=i A .
$$

As a result, as we are considering the case of a finite dimensional Hilbert space it is immediate to prove that the space $\mathcal{O}$ may be identified also with the space $\mathfrak{u}^{*}(\mathcal{H})$. We summarize this result in the form:

Lemma 1. The space of observables $\mathcal{O}$ becomes endowed with a Lie algebra structure, isomorphic to the natural structure on $\mathfrak{u}(\mathcal{H})$ by defining

$$
[A, B]_{-}=\alpha^{-1}(\alpha(A) \alpha(B)-\alpha(B) \alpha(A))=-i[\alpha(A), \alpha(B)],
$$

along with a real scalar product that is invariant under the adjoint representation, given by

$$
\langle A, B\rangle=\frac{1}{2} \operatorname{Tr}(A B) .
$$

Another isomorphism can be defined identifying the vector spaces $\mathfrak{u}(\mathcal{H})$ and $\mathfrak{u}^{*}(\mathcal{H})$, according to the pairing given by the Killing-Cartan form

$$
\xi(A)=\frac{i}{2} \operatorname{Tr}(\xi A) \quad \xi \in \mathfrak{u}^{*}(\mathcal{H}), A \in \mathfrak{u}(\mathcal{H})
$$

The space of Hermitian operators carries also another binary product (usually called Jordan product) defined by

$$
A \circ B=\frac{1}{2}(A B+B A)=[A, B]_{+} .
$$

Let us recall, for completeness, the definition of Jordan algebra: 
Definition 1. A non-associative algebra $(\mathcal{A}, \cdot)$ is called a Jordan algebra if and only if, the operation is commutative and given two arbitrary elements $x, y \in \mathcal{A},(x y) x^{2}=x\left(y x^{2}\right)$

With this definition we can conclude

Lemma 2. $(\mathcal{O}, \circ)$ is a Jordan algebra.

Proof. The commutativity is trivial to prove. The second condition follows from the associativity of the original product:

$$
\begin{aligned}
& {\left[[x, y]_{+}, x^{2}\right]_{+}=2(x y+y x) x^{2}+2 x^{2}(x y+y x)=2\left(x y x^{2}+y x^{3}+x^{3} y+x^{2} y x\right)} \\
& {\left[x,\left[y, x^{2}\right]_{+}\right]_{+}=2 x\left(y x^{2}+x^{2} y\right)+\left(y x^{2}+x^{2} y\right) x=2\left(x y x^{2}+x^{3} y+y x^{3}+x^{2} y x\right)}
\end{aligned}
$$

Proposition 1. The scalar product (4) is also invariant with respect to this new product, and we have:

$$
\left\langle[A, B]_{-}, C\right\rangle=\left\langle A,[B, C]_{-}\right\rangle, \quad\left\langle[A, B]_{+}, C\right\rangle=\left\langle A,[B, C]_{+}\right\rangle .
$$

Moreover we also have the compatibility relation

$$
[A, B \circ C]_{-}=[A, B]_{-} \circ C+B \circ[A, C]_{-}
$$

i.e. ad_ is a derivation of the Jordan algebra.

Proof. These properties follow directly from the definitions.

From [18, 17], we define further

Definition 2. A Lie algebra $\left(\mathcal{A},[\cdot, \cdot]_{-}\right)$is called a Jordan-Lie algebra if it admits a Jordan algebra structure o satisfying (8) and the associator identity, for some $\hbar \in \mathbb{R}$

$$
(A \circ B) \circ C-A \circ(B \circ C)=\frac{1}{4} \hbar\left[[A, C]_{-}, B\right]_{-} \quad \forall A, B, C \in \mathcal{A}
$$

Lemma 3. The space of observables, with the operations defined above, becomes also a Jordan-Lie algebra.

Proof. See [17] for details. 


\subsection{The geometrical structures}

A specific way to geometrize the Lie algebra structure of $\mathfrak{u}(\mathcal{H})$ is to associate with it a linear of Poisson tensor on the dual vector space $\mathfrak{u}^{*}(\mathcal{H})$ as follows. As $\mathcal{H}$ is assumed to be finite-dimensional, we can identify $\mathfrak{u}(\mathcal{H})$ with the space of real valued linear functions on its dual space, i.e. $\mathfrak{u}(\mathcal{H}) \sim \operatorname{Lin}\left(\mathfrak{u}^{*}(\mathcal{H}), \mathbb{R}\right)$, and we set, for any pair of linear functions on $\mathfrak{u}^{*}(\mathcal{H})$ defined by the two elements $u, v \in \mathfrak{u}(\mathcal{H})$ :

$$
\{\hat{u}, \hat{v}\}=\widehat{[u, v]},
$$

where the commutator on the right hand side is computed by thinking of $u, v$ as elements of the Lie algebra $\mathfrak{u}(\mathcal{H})$, and the left hand side is to be read as a linear function on $\mathfrak{u}^{*}(\mathcal{H})$. We will use the 'hat' to denote the elements of $\mathfrak{u}(\mathcal{H})$ seen as linear functions on the dual $\mathfrak{u}^{*}(\mathcal{H})$. We are implicitly using here the property that the vector space $\mathfrak{u}(\mathcal{H})$ is isomorphic to its bidual, which holds for vector spaces which are reflexive, in particular finite dimensional ones. Then, we have:

Proposition 2. Let $\mathcal{O}$ be the space of observables of a finite level quantum system. Then, $\mathcal{O}^{*}$ (and $\mathcal{O}$ itself) can be endowed with a Poisson structure.

Having replaced the Lie algebra structure with the Poisson tensor associated with the Poisson bracket on $\mathfrak{u}^{*}(\mathcal{H})$, we are now able to perform also nonlinear transformations on the Poisson manifold. In this sense we speak of the 'geometrization' of the algebra structure of the vector space $\mathfrak{u}(\mathcal{H})$.

If we denote by $\hat{A}$ and $\hat{B}$ the linear functions on $\mathfrak{u}^{*}(\mathcal{H})$ corresponding to elements $A, B \in \mathfrak{u}(\mathcal{H})$, we can define the Poisson bivector $\Lambda$ as:

$$
\Lambda(d \hat{A}, d \hat{B})(\xi)=\{\hat{A}, \hat{B}\}(\xi)=\xi([A, B])=\frac{i}{2} \operatorname{Tr} \xi(A B-B A), \quad \xi \in \mathfrak{u}^{*}(\mathcal{H})
$$

where we used the relation (5).

Hence we recover the well-known Kirillov-Konstant-Souriau Poisson tensor on the dual of any Lie algebra.

By using a similar procedure we may also 'geometrize' the Jordan algebra structure on the space of observables. Again we set:

$$
\mathcal{R}(\xi)(d \hat{A}, d \hat{B})=\xi\left([A, B]_{+}\right)=\frac{1}{2} \operatorname{Tr} \xi(A B+B A), \quad \xi \in \mathfrak{u}^{*}(\mathcal{H}),
$$

where use is made of (5). 
These two tensor fields can be put together to form a complex vector field:

$$
(\mathcal{R}+i \Lambda)(\xi)(d \hat{A}, d \hat{B})=2(\widehat{A B})(\xi)=\xi(A B)=\operatorname{Tr}(\xi A B), \quad \xi \in \mathfrak{u}^{*}(\mathcal{H})
$$

By using this tensor field we can define a $*$-product in the form

$$
(\hat{A} * \hat{B})(\xi)=\xi(A B)=(\mathcal{R}+i \Lambda)(\xi)(d \hat{A}, d \hat{B}) .
$$

In this context, the compatibility condition of the Lie and the Jordan structures can be simply stated by saying

Proposition 3. The Hamiltonian vector fields associated with observables (i.e. linear functions on $\mathfrak{u}^{*}(\mathcal{H})$ ) are infinitesimal symmetries for the tensor field $\mathcal{R}$ associated with the Jordan structure, and therefore derivations for the *-product.

Proof. It is a direct consequence of the compatibility between both brackets, summarized in (8).

\subsection{Distributions}

In this section we will summarize very quickly the results presented in [20, 21], regarding the definition of suitable distributions defined on $\mathfrak{u}(\mathcal{H})$ which correspond to the geometric objects defined above. By using the identification of the real vector space $\mathfrak{u}(\mathcal{H})$ with its tangent space at each point, we may define a $(1,1)$-tensor field $\hat{J}$ in $\mathfrak{u}(\mathcal{H})$ by setting

$$
\hat{J}_{\xi}(A)=[A, \xi]_{-}=\Lambda_{\xi}(d \hat{A}), \quad A \in \mathfrak{u}(\mathcal{H}) .
$$

Similarly we can write:

$$
\hat{\mathcal{R}}_{\xi}(A)=[A, \xi]_{+}=\mathcal{R}_{\xi}(d \hat{A}), \quad A \in \mathfrak{u}(\mathcal{H}) .
$$

These two tensors allow us to define two important distributions:

$$
D_{\Lambda}=\operatorname{Image}(\hat{J}), \quad D_{\mathcal{R}}=\operatorname{Image}(\hat{\mathcal{R}})
$$

Considering these two objects we find that both tensors commute among themselves:

$$
\hat{J} \circ \hat{\mathcal{R}}(A)=\hat{\mathcal{R}} \circ \hat{J}(A)=\frac{1}{2}\left[A, \xi^{2}\right]_{-} .
$$

Both distributions can be combined into two new ones: $D_{0}=D_{\mathcal{R}} \cap D_{\Lambda}$ and $D_{1}=D_{\mathcal{R}}+D_{\Lambda}$. 
Proposition 4. The distributions $D_{0}, D_{1}, D_{\mathcal{R}}$ and $D_{\Lambda}$ satisfy ([20]):

- $D_{\Lambda}$ is an involutive distribution providing a generalized foliation whose leaves are the symplectic manifolds associated with the Poisson tensor $\Lambda$.

- The distribution $D_{\mathcal{R}}$ is not involutive.

- The distribution $D_{0}$ is involutive. The corresponding foliation is invariant under the adjoint representation and each leaf is a Kähler manifold.

- The distribution $D_{1}$ is involutive and the corresponding leaves of its associated foliation are orbits of the $\mathrm{GL}(\mathcal{H})$ action defined as

$$
\mathrm{GL}(\mathcal{H}) \times \mathfrak{u}(\mathcal{H}) \rightarrow \mathfrak{u}(\mathcal{H}), \quad(T, A) \mapsto T A T^{\dagger} .
$$

Remark 1. Our 'geometrization' carries along the possibility of performing nonlinear transformations because we have replaced the algebraic structures on the linear space $\mathfrak{u}(\mathcal{H})$ with tensorial objects on the manifold $u^{*}(\mathcal{H})$. It should be remarked, however, that now on $\mathfrak{u}^{*}(\mathcal{H})$ we have the possibility of two different products on linear functions:

- the pointwise product $(\hat{A} \cdot \hat{B})(\xi)=\hat{A}(\xi) \hat{B}(\xi)$, which gives a quadratic function out of two linear ones and

- a non-local product $(\hat{A} \star \hat{B})(\xi)=\widehat{A B}(\xi)$. In this case we obtain a linear function as the product of other two linear ones, but in general it will be a complex valued function even if the factors were real ones. This result has to do with the fact that the product of two Hermitian operators is not Hermitian and therefore it gives rise to real and imaginary parts.

Example 1. At this point it may be adequate to give a simple example of the objects introduced so far. Let us consider the Lie algebra $\mathfrak{s u}(2)$ of $2 \times 2 \mathrm{Her}$ mitian matrices corresponding to a spin 1/2 physical system. We introduce an orthonormal basis with respect to the scalar product (41). We set thus:

$$
U=\left(\begin{array}{ll}
1 & 0 \\
0 & 1
\end{array}\right), \quad X=\left(\begin{array}{cc}
0 & 1 \\
1 & 0
\end{array}\right), \quad Y=\left(\begin{array}{cc}
0 & -i \\
i & 0
\end{array}\right), \quad Z=\left(\begin{array}{cc}
1 & 0 \\
0 & -1
\end{array}\right)
$$

and also the associated linear functions

$$
\hat{X}=x, \quad \hat{Y}=y, \quad \hat{Z}=z, \quad \hat{U}=u
$$


where the functions are to be understood as $z(A)=\frac{i}{2} \operatorname{Tr}(Z A)$, and so on, for any $A \in u(2)$. In these coordinates, the Poisson tensor field is given by

$$
\Lambda=2\left(x \frac{\partial}{\partial y} \wedge \frac{\partial}{\partial z}+y \frac{\partial}{\partial z} \wedge \frac{\partial}{\partial x}+z \frac{\partial}{\partial x} \wedge \frac{\partial}{\partial y}\right)
$$

while the tensor associated to the Jordan structure becomes:

$$
\begin{aligned}
\mathcal{R} & =2 \frac{\partial}{\partial u} \otimes_{s}\left(x \frac{\partial}{\partial x}+y \frac{\partial}{\partial y}+z \frac{\partial}{\partial z}\right) \\
& +2 u\left(\frac{\partial}{\partial u} \otimes_{s} \frac{\partial}{\partial u}+\frac{\partial}{\partial x} \otimes_{s} \frac{\partial}{\partial x}+\frac{\partial}{\partial y} \otimes_{s} \frac{\partial}{\partial y}+\frac{\partial}{\partial z} \otimes_{s} \frac{\partial}{\partial z}\right)
\end{aligned}
$$

where $\otimes_{s}$ stands for the symmetrized tensor product.

It is immediately seen that $\mathcal{R}$ is invariant under the rotation vector fields provided by the linear Hamiltonian functions with respect to the Poisson tensor $\Lambda$. We can even consider the non-local product, for instance we get

$$
\hat{Z} \star \hat{Y}=-i \hat{X}, \quad \hat{X} \star \hat{Y}=i \hat{Z}, \quad \hat{Z} \star \hat{X}=i \hat{Y} .
$$

It is also easy to see that the Hamiltonian vector fields associated with linear functions provide derivations both for the pointwise product and for the non-local product. Thus, the associated equations of motion do not carry a quantum or a classical label, it is the product what distinguishes the commutative or the non-commutative nature of the space along with the locality or non-locality of the operation. And therefore distinguishes Classical from Quantum Mechanics.

\subsection{Dynamics}

It is now possible to write equations of motion on the phase space of observables. In the pure Heisenberg picture it is defined as

$$
\frac{d}{d t} A=\frac{1}{\hbar}[H, A]_{-} .
$$

By using the 'geometrization', i.e. by thinking in terms of the dual space $\mathfrak{u}^{*}(\mathcal{H})$, we find

$$
\frac{d}{d t} \widehat{A}=\frac{1}{\hbar}\{\widehat{H}, \widehat{A}\} .
$$

As there are different algebra structures on $\mathfrak{u}^{*}(\mathcal{H})$ it is important to study the compatibility of differential equations with such algebra structures. 
Lemma 4. The linear differential equations which preserve both products correspond to the infinitesimal generators of unitary transformations.

\subsection{States}

States are identified as the elements of the convex body $\mathcal{S}=\left\{\phi \in \mathcal{A}^{*}\right.$ $\left.\phi\left(A^{*} A\right) \geq 0, \forall A \in \mathcal{A} ; \phi(\mathbb{I})=1\right\}$.

It is not difficult to show in the finite-dimensional case, for the infinitedimensional case it is a theorem by Gleason, that any state can be written in the form $\phi(A)=\operatorname{Tr} \rho_{\phi} A$.

Moreover, $\rho_{\phi}$ is a nonegatively defined operator of $\mathfrak{g l}(\mathcal{H})$, i.e. those $\rho_{\phi} \in$ $\mathfrak{g l}(\mathcal{H})$ which can be written in the form $\rho_{\phi}=T^{\dagger} T$ for some $T \in \mathfrak{g l}(\mathcal{H})$ and, in addition, satisfy $\operatorname{Tr} \rho_{\phi}=1$. Thus, $\mathcal{S}$ is a convex body in the affine hyperplane in $\mathfrak{u}^{*}(\mathcal{H})$, determined by the equation $\operatorname{Tr} \rho_{\phi}=1$. The tangent space to this affine hyperplane at a point is therefore identified with the space of traceless Hermitian operators, and it is in a one-to-one correspondence with the Lie algebra of the group $S U(\mathcal{H})$.

\section{Symplectic realizations of the Poisson man- ifold $\mathfrak{u}^{*}$}

\subsection{The representation in the finite dimensional case}

In the general case, to reconstruct a Hilbert space representation from the 'algebraic description' of quantum systems, along with its 'geometrization', we can use the Gelfand-Naimark-Segal (GNS) construction. This requires the introduction of a state (a functional on the algebra of observables which satisfies certain conditions) and the definition of an adapted Hilbert space where the algebra of observables can be represented.

In this finite-dimensional situation there is a simpler alternative. We may use techniques from differential geometry of Hamiltonian systems to construct a representation on a Hilbert space. Specifically, we search for a symplectic realization of the Poisson manifold by means of a symplectic vector space (a classical Jordan-Schwinger map)

Definition 3. A symplectic realization of a Poisson manifold $(N,\{\cdot, \cdot\})$ is a Poisson map $\Phi: M \rightarrow N$, where $(M, \omega)$ is a symplectic manifold. When 
$M$ is a symplectic vector space we have a special situation and $\Phi$ is called a classical Jordan-Schwinger map [19].

\subsubsection{The Kähler space}

The first step is to consider the dimension of the algebra of observables. From the dimension of $\mathfrak{u}(\mathcal{H})$, say $n^{2}$-dimensional, we may consider the action of the unitary group $U(n)$ on the vector space $\mathbb{R}^{2 n}$, endowed with a Kähler structure, a triple $(\omega, g, J)$. Here $\omega$ is an exact symplectic structure, $g$ is an Euclidean structure and $J$ the associated complex structure. We consider what is known as the "defining representation" of the group $U(n)$.

The symplectic structure admits a potential 1-form which is invariant under the action and then the associated momentum map provides us with the symplectic realization we are searching for (see [22]). The momentum map, $\mu: \mathbb{R}^{2 n} \rightarrow \mathfrak{u}^{*}(n)$, is equivariant with respect to the fundamental action of $U(n)$ on $\mathbb{C}^{n} \approx \mathbb{R}^{2 n}$ and the coadjoint action on $\mathfrak{u}^{*}(n)$.

If we select an orthonormal basis $\left\{e_{1}, \ldots, e_{n}\right\}$ for $\mathbb{C}^{n}$, we may define coordinates by setting $\left\langle e_{k} \mid \psi\right\rangle=z_{k}(\psi)=\left(q_{k}+i p_{k}\right)(\psi)$, and we have used Dirac's notation for bras and kets.

\subsubsection{The geometric structures}

One can see $([20])$ that in these coordinates we have a contravariant version of the Euclidean structure given by $G=\sum_{k=1}^{n}\left(\frac{\partial}{\partial q_{k}} \otimes \frac{\partial}{\partial q_{k}}+\frac{\partial}{\partial p_{k}} \otimes \frac{\partial}{\partial p_{k}}\right)$ and the Poisson tensor $\Omega=\sum_{k=1}^{n}\left(\frac{\partial}{\partial q_{k}} \wedge \frac{\partial}{\partial p_{k}}\right)$ while the complex structure has the form $J=\sum_{k=1}^{n}\left(\frac{\partial}{\partial p_{k}} \otimes d q_{k}+\frac{\partial}{\partial q_{k}} \otimes d p_{k}\right)$.

In terms of complex coordinates the Hermitian structure has the form $h=\sum_{k=1}^{n} d \bar{z}_{k} \otimes d z_{k}$. The corresponding contravariant form is given by

$$
G+i \Omega=\sum_{k=1}^{n}\left(\frac{\partial}{\partial q_{k}}-i \frac{\partial}{\partial q_{k}}\right) \otimes\left(\frac{\partial}{\partial q_{k}}+i \frac{\partial}{\partial q_{k}}\right)=4 \sum_{k=1}^{n} \frac{\partial}{\partial z_{k}} \otimes \frac{\partial}{\partial \bar{z}_{k}}
$$


We may define binary products on functions by setting

$$
\begin{aligned}
\left\{f_{1}, f_{2}\right\} & =\sum_{k=1}^{n}\left(\frac{\partial f_{1}}{\partial q_{k}} \frac{\partial f_{2}}{\partial p_{k}}-\frac{\partial f_{1}}{\partial p_{k}} \frac{\partial f_{2}}{\partial q_{k}}\right) \\
\left\{f_{1}, f_{2}\right\}_{+} & =\sum_{k=1}^{n}\left(\frac{\partial f_{1}}{\partial q_{k}} \frac{\partial f_{2}}{\partial p_{k}}+\frac{\partial f_{1}}{\partial p_{k}} \frac{\partial f_{2}}{\partial q_{k}}\right) \\
\left\langle f_{1} \mid f_{2}\right\rangle & =4 \sum_{k=1}^{n} \frac{\partial f_{1}}{\partial z_{k}} \frac{\partial f_{2}}{\partial \bar{z}_{k}}
\end{aligned}
$$

\subsection{The representation of $\mathcal{O}$}

Now we consider the corresponding representation of the algebra of observables. First we can consider the following trivial lemma:

Lemma 5. Every complex linear operator $A \in \mathfrak{g l}(n, \mathbb{C})$ defines a quadratic function $f_{A}(\psi)=\frac{1}{2}\langle\psi \mid A \psi\rangle$. The function is real if and only if $A$ is Hermitian, $A=A^{\dagger}$.

Having introduced the relevant geometrical structures associated with the Kähler structure, we may now proceed to establish the connection between the description on the space of observables, $\mathfrak{u}(\mathcal{H})$, with the description on the Hilbert space $\mathcal{H} \equiv \mathbb{C}^{n}$.

Proposition 5. The main properties of the momentum map,

$$
\mu: \mathcal{H} \rightarrow \mathfrak{u}^{*}(\mathcal{H}), \quad \mu:|\psi\rangle \mapsto \rho_{\psi}=|\psi\rangle\langle\psi|,
$$

are the following:

- $\mu^{*}(\widehat{A})=f_{A}$

- $\mu^{*}(\{\widehat{A}, \widehat{B}\})=\left\{f_{A}, f_{B}\right\}$

- $\mu^{*}(\mathcal{R}(d \widehat{A}, d \widehat{B}))=G\left(\mu^{*}(d \widehat{A}), \mu^{*}(d \widehat{B})\right)$

Proof. Direct verification.

Thus we conclude that the geometrization of the observables on the Hilbert space corresponds to the study of the associated quadratic functions. 


\subsection{Dynamics}

It is now possible to write the dynamics on the Hilbert space, Schrödinger equation, by writing $i \hbar \frac{d}{d t}|\psi\rangle=H|\psi\rangle$ or equivalently, $\hbar \frac{d}{d t} f_{A}=\left\{f_{H}, f_{A}\right\}$.

This last equation is $\mu$-related with the Heisenberg equation on the space of observables.

\subsection{Eigenvalues and eigenstates}

In the 'geometrized' Hilbert space description we have to recover now the description of the 'eigenvectors' and 'eigenvalues'.

To this aim is appropriate to introduce expectation values associated with Hermitian operators, $A=A^{\dagger}$ :

$$
e_{A}(\psi)=\frac{\langle\psi \mid A \psi\rangle}{\langle\psi \mid \psi\rangle} .
$$

We find that:

1. Critical points of $d e_{A}$ correspond to the eigenvectors of $A$.

2. Values of $e_{A}$ at critical points are the corresponding eigenvalues of $A$.

Remark 2. Critical points of de $A_{A}$ coincide with the critical points of the corresponding Hamiltonian vector field $\Omega\left(d e_{A}\right)$ or of the corresponding gradient vector field $G\left(d e_{A}\right)$.

\section{Remark 3.}

$$
G\left(d e_{A}, d e_{A}\right)=\frac{\left\langle\psi \mid A^{2} \psi\right\rangle}{\langle\psi \mid \psi\rangle}-\frac{\langle\psi \mid A \psi\rangle}{\langle\psi \mid \psi\rangle} \frac{\langle\psi \mid A \psi\rangle}{\langle\psi \mid \psi\rangle} .
$$

i.e. it represents the dispersion of the mean value of the observable corresponding to the operator $A$ in the state $|\psi\rangle$, therefore the square of the Hamiltonian vector field associated with $A$ is strictly related to the 'uncertainty' in the measurement of $A$ in the state $|\psi\rangle$.

Remark 4. The GNS construction associated with a pure state would provide us with a complex Hilbert space of dimension $n$ if we start with $n \times n$ Hermitian matrices. Therefore, it may be considered as a way to construct a "symplectic realization" of the algebraic structures on $\mathfrak{u}^{*}(\mathcal{H})$ (which also realizes the Jordan algebra). 


\section{Conclusions}

In this paper we have presented a way of providing a geometrization of Quantum Mechanics starting from the set of observables of our quantum system. To close the circle, we may try to connect our results with the usual geometrical constructions which take the space of states as starting point.

The probabilistic interpretation of quantum mechanics requires the states to be normalized and, moreover, because normalized vectors are identified with probability amplitudes, the physically relevant probability densities are invariant under multiplication of vectors by a phase. In conclusion, physical states should be identified not with vectors in the Hilbert space but rather with points in the associated complex projective space. We thus define the principal fibration $\mathbb{C}_{0} \rightarrow \mathcal{H}-\{0\} \rightarrow \mathbb{P} \mathcal{H}$, where $\mathbb{C}_{0}=\mathbb{C}-\{0\}$ is the set of nonzero complex numbers and $\mathcal{H}-\{0\}$ that of nonzero vectors. $\mathbb{P} \mathcal{H}$, denotes the complex projective space associated with $\mathcal{H}$.

From the manifold point of view the quotient $\mathbb{P} \mathcal{H}$ is completely described by the involutive distribution generated by $\Delta$, the infinitesimal generator of dilations, and by $J(\Delta)$, the infinitesimal generator of multiplication by a phase. They provide enough information to describe the points of the projective space. The relevant tensors $G$ and $\Omega$ introduced in the previous section, however, are not projectable with respect to the fibration above. To turn them into projectable tensors we can multiply them by a conformal factor $\langle\psi \mid \psi\rangle$ and in this way we get projectable tensor fields. However, $\langle\psi \mid \psi\rangle \Omega$ does not define a bracket satisfying the Jacobi identity. As a matter of fact, it gives rise to a Jacobi bracket. Therefore, the Poisson bracket we obtain on the complex projective space has to be considered as a reduction of a Jacobi bracket on $\mathcal{H}$ instead of a Poisson bracket on $\mathcal{H}$. Further details can be found in 15 .

To close the circle, we notice that the complex projective space may be identified with minimal symplectic orbits on $\mathfrak{u}^{*}(\mathcal{H})$ (see [20]). The Schrödinger equations of motion on the Hilbert space project onto von Neumann equations of motion on the space of pure states (rank one projectors). See [23].

With this observation we conclude by saying that (all) various formalisms to describe quantum systems may be 'geometrized' and these 'geometrizations' rely on the consideration of the momentum map associated with the

strongly Hamiltonian action of the unitary group on the Hilbert space of states. The treatment has been carried out for finite level quantum systems. However the extension to infinite-dimensional Hilbert spaces encounters only 
problems we can call technical, the conceptual geometrical framework is fully captured by the finite dimensional situation.

\section{References}

[1] G. Marmo and G. Morandi, Some Geometry and Topology in Low dimensional Quantum Field Theory for condensed matter physicists, Lecture Notes of ICTP,1-108, Trieste (1992)

[2] P.A.M. Dirac, The Principles of Quantum Mechanics, Clarendon Press, Oxford, 2nd edition (1936).

[3] E. Segal, "Postulates for general quantum mechanics", Annals of Math. 48, 930-948 (1947).

[4] R. Haag and D. Kastler, "An algebraic approach to quantum field theory", J. Math. Phys. 5, 848-861 (1964).

[5] R. Cirelli, P. Lanzavecchia and A. Maniá, Normal pure states of the von Neumann algebra of bounded operator as Kähler manifold, J. Phys. A: Math. Gen. 15, 3829-3835 (1983)

[6] R. Cirelli and P. Lanzavecchia, Hamiltonian vector fields in Quantum Mechanics, Nuovo Cimento B, 79, 271-283 (1984)

[7] M.C. Abbati, R. Cirelli, P. Lanzavecchia and A. Maniá, Pure states of general quantum mechanical systems as Kähler bundle, Nuovo Cimento B, 83, 43-60 (1984)

[8] A. Bloch, An infinite-dimensional Hamiltonian system on a projective Hilbert space, Trans. AMS 302, 787-796 (1987)

[9] A. Heslot, Quantum mechanics as a classical theory, Phys Rev D 31 , $1341-1348$ (1985)

[10] D.J. Rowe, A. Ryman and G. Rosensteel, Many body quantum mechanics as a symplectic dynamical system, Phys Rev A 22, 2362-2372 (1980)

[11] T.R. Field and J.S. Anandan, Geometric phases and coherent states, J. Geom. Phys. 50, 56-78 (2004) 
[12] D. Brody and L.P. Hughston, Geometric quantum mechanics, J. Geom. Phys. 38, 19-53 (2001)

[13] F. Strocchi, Complex coordinates and quantum mechanics, Rev. Mod. Phys. 38, 36-40 (1956)

[14] A. Ashtekar and T.A. Shilling, Geometrical formulation of Quantum Mechanics, in On Einstein's path, Ed. A. Harvey, Springer (1998)

[15] V. I. Manko, G. Marmo, E.C.G. Sudarshan and F. Zaccaria, The geometry of density states, Rep Math Phys 55, 405-422 (2005)

[16] P. Jordan, J. von Neumann and E.P. Wigner, "On an algebraic generalization of the quantum mechanical formalism", Ann. Math. 35, 29-64 (1934)

[17] G.G. Emch, Foundations of 20th century Physics, North Holland, Amsterdam (1984)

[18] N. P. Landsman, Mathematical Topics Between Classical and Quantum Mechanics, Springer Mathematical Monographs, Springer, New York, 1998

[19] V.I. Manko, G. Marmo, P. Vitale and F. Zaccaria, A generalization of the Jordan-Schwinger map: classical version and its q-deformation, Int. J. Mod. Phys. A, 9, 5541-5561 (1994)

[20] J. Grabowski, M. Kuś and G. Marmo, "Geometry of quantum systems: density states and entanglement", J. Phys. A:Math. Gen 38, 1021710244 (2005).

[21] J. Grabowski, M. Kuś and G. Marmo, "Symmetry, group actions and entanglement", Open sys. \& Information dyn. (2006, to appear).

[22] L.J. Boya, J.F. Cariñena and J.M. Gracia-Bondía, Symplectic structure of the Aharonov-Anandan geometric phase, Phys. Lett. A 161, 30-34 (1991).

[23] V.I. Man'ko, G. Marmo, E.C.G. Sudarshan and F. Zaccaria, "On the relation between Schrödinger and von Neumann equations", J. Russ. Laser Research 20, 421-437 (1999) 